\title{
Performance Evaluation of Safety Applications over DSRC Vehicular Ad Hoc Networks
}

\author{
Jijun Yin Tamer ElBatt \\ Gavin Yeung Bo Ryu \\ Information Sciences Laboratory \\ HRL Laboratories, LLC \\ Malibu, CA 90265, USA \\ jijun@hrl.com,telbatt@hrl.com
}

\author{
Stephen Habermas \\ Hariharan Krishnan \\ Timothy Talty \\ General Motors Corporation \\ Warren, MI 48090, USA
}

\begin{abstract}
In this paper we conduct a feasibility study of delay-critical safety applications over vehicular ad hoc networks based on the emerging dedicated short range communications (DSRC) standard. In particular, we quantify the bit error rate, throughput and latency associated with vehicle collision avoidance applications running on top of mobile ad hoc networks employing the physical and MAC layers of DSRC. Towards this objective, the study goes through two phases. First, we conduct a detailed simulation study of the DSRC physical layer in order to judge the link bit error rate performance under a wide variety of vehicles speeds and multi-path delay spreads. We observe that the physical layer is highly immune to large delay spreads that might arise in the highway environment whereas performance degrades considerably at high speeds in a multi-path environment. Second, we develop a simulation testbed for a DSRC vehicular ad hoc network executing vehicle collision avoidance applications in an attempt to gauge the level of support the DSRC standard provides for this type of applications. Initial results reveal that DSRC achieves promising latency performance, yet, the throughput performance needs further improvement.
\end{abstract}

\section{Categories and Subject Descriptors}

C.2.5 [Computer-Communication Networks]: Local and WideArea Networks; C.2.1 [Computer-Communication Networks]: Network Architecture and Design-Wireless communication

\section{General Terms}

Performance, Theory

\section{Keywords}

Intervehicle communications, MANETs, DSRC, bit error rate, safety applications, simulation, OFDM, throughput, latency.

Permission to make digital or hard copies of all or part of this work for personal or classroom use is granted without fee provided that copies are not made or distributed for profit or commercial advantage and that copies bear this notice and the full citation on the first page. To copy otherwise, to republish, to post on servers or to redistribute to lists, requires prior specific permission and/or a fee.

VANET'04, October 1, 2004, Philadelphia, Pennsylvania, USA.

Copyright 2004 ACM 1-58113-922-5/04/0010 ...\$5.00.

\section{INTRODUCTION}

The rapid evolution of wireless data communications technologies witnessed recently creates ample opportunity to utilize these technologies in support of vehicle safety applications. At one hand, Cellular-based systems (e.g. OnStar) [1] have contributed the ability to report accidents in a timely and reliable manner. On the other hand, new wireless technologies have the potential to enable intervehicle communications for the purpose of crash avoidance. Mobile ad hoc networking (MANET) is a potential technology for supporting these applications in a secure, resource-efficient, and reliable manner. In addition, the DSRC standard at $5.9 \mathrm{GHz}$ band is projected to support low-latency wireless data communications between vehicles and from vehicles to roadside units. The DSRC specification [2] is meant to be an extension of the IEEE 802.11 [3] technology into the outdoor high-speed vehicle environment. In fact, the physical layer (PHY) of DSRC is adapted from IEEE 802.11a PHY based on orthogonal frequency division multiplex (OFDM) technology. Moreover, the multiple access control (MAC) layer of DSRC is very similar to the IEEE 802.11 MAC based on the carrier sense multiple access with collision avoidance (CSMA/CA) protocol with some minor modifications.

Inter-vehicle communications open the door for a plethora of applications and services ranging from automated highway systems [4] to distributed passengers teleconference. These applications may be classified to safety and non-safety applications. Under safety applications, Vehicle Collision Avoidance (VCA) has attracted considerable attention since it is directly related to minimizing number of accidents on the road. On the other hand, non-safety applications may include real-time road traffic estimation for trip planning, high-speed tolling, collaborative expedition, information retrieval, and entertainment applications. In addition, we envision that vehicular ad hoc networks (VANET) may play an important role in improving the capacity and coverage of future wireless networks via: i) Complementing the existing cellular infrastructure in hot spot areas where the system gets overloaded and it may be favorable for vehicles to assist one another in reaching the base station (BS) (via multi-hopping) rather than continously competing to access the uplink and ii) Extending the coverage of the cellular infrastructure via enabling an out-of-range vehicle to forward its data through multiple hops until a BS is reachable.

In [5], the authors outline a number of challenges introduced by the DSRC operating environment to IEEE 802.11 MAC, namely multi-hop operation, high mobility and QoS support. The study in [6] introduces a location-based broadcast communication protocol for supporting safety applications in the $5.9 \mathrm{GHz}$ band. However, the wireless propagation model does not account for the multi-path 
delay spread and Doppler effect which may have significant impact on performance. In addition, the vehicle mobility model underlying this study is not provided in the paper. A communication protocol for low-latency delivery of emergency warnings under various road situations has been investigated in [7]. The study in [8] analyzes the packet error rate performance of the IEEE 802.11a/RA PHY (variant of the IEEE wireless LAN standard developed for Roadside Applications) in the outdoor high-speed vehicular communication environment. The results reveal high packet error rate which confirms the significant impact the physical layer performance will have on the end-to-end VANET performance in such harsh wireless environment. In [9], the authors introduce a distributed wireless token ring MAC protocol in an attempt to guarantee bounded delay and achieve fairness in networks of vehicular platoons. Similarly, [10] presents a collision-free medium access control mechanism based on vehicles' location information in order to support the delay-bounded requirement dictated by safety applications. The simulation study in [11] shows the benefits of multi-hopping over alternate paths when direct communication between vehicles can not be achieved. In [12], the authors introduced an approach for disseminating safety messages among highly mobile hosts. Finally, [13] discusses the rationale behind choosing position-based routing [14] and UTRA-TDD [15] MAC protocols, among other alternatives, as candidate protocols for the Fleetnet project [16]. The common limitation among $[7,9,10,11,12,16]$ is that the physical layer model was highly abstract and did not account for multi-path delay spread and Doppler effect. This, in turn, motivated us to conduct a VANET simulation study that incorporates a detailed PHY model of DSRC.

Our contribution in this paper is two-fold: i) Quantify the physical layer performance of the current DSRC standard, measured in bit error rate (BER), under large Doppler and multi-path delay spread encountered in the outdoor high-speed vehicle environment and ii) Characterize the throughput and latency performance of vehicle collision avoidance applications over a DSRC VANET. First, we develop a detailed physical layer simulation for the DSRC standard which accurately models the vehicle-to-vehicle wireless link propagation at $5.9 \mathrm{GHz}$. Next, we incorporate the BER results obtained earlier into a VANET simulation testbed that analyzes the performance of vehicle collision avoidance applications in light of the quality of service (QoS) requirements provided by the Vehicle Safety Communications Consortium (VSCC) [19].

The paper is organized as follows: In section II, we introduce a brief overview of the DSRC standard. Afterwards, the DSRC PHY layer performance evaluation study is conducted in section III. This is followed by analyzing the performance of vehicle collision avoidance applications over DSRC VANETs in section IV. Finally, conclusions are drawn in section V.

\section{DSRC OVERVIEW}

In October 1999, the Federal Communications Commission (FCC) allocated the $5.9 \mathrm{GHz}$ band for DSRC-based intelligent transportation systems (ITS) applications and adopted basic technical rules for DSRC operation. On July 10, 2003, the standards writing group (an ASTM working group) approved the ASTM-DSRC Standard for DSRC operations. This standard is based on IEEE 802.11a physical layer and IEEE 802.11 MAC layer and was published as ASTM E2213-03 [2] in Sep. 2003. FCC report and order, issued in Feb. 2004, has established service and licensing rules to govern the use of the DSRC band. In addition, it adopted [2] to ensure the inter-operability and robust safety/public safety communications among these DSRC devices nationwide. Currently, the ASTM E2213-03 standard is being migrated to the IEEE 802.11 standard where it is undergoing revision by the newly formed WAVE study group within the IEEE 802.11 community.

The 5.9Ghz band consists of seven ten-megahertz channels which includes one control channel and six service channels. DSRC, which involves vehicle-to-vehicle and vehicle-to-infrastructure communications, is expected to support both safety/public safety and non-safety applications. However, priority is given to safety applications since the non-public safety use of the $5.9 \mathrm{GHz}$ band would be inappropriate if it leads to degrading the performance of safety/public safety applications [17]. This is attributed to the fact that safety applications are meant to save lives via warning drivers of an impending dangerous condition or event in a timely manner in order to take corrective actions. Therefore, response time and reliability are basic requirements of safety applications as discussed later in the paper.

DSRC PHY uses OFDM modulation scheme to multiplex data. Along with the successful deployment of IEEE 802.11a WLAN services and devices in recent years, OFDM has gained increased popularity in the wireless communication community due to its high spectral efficiency, inherent capability to combat multi-path fading and simple transceiver design. In a nut shell, the input data stream is divided into a set of parallel bit streams and each bit stream is then mapped onto a set of overlapping orthogonal subcarriers for data modulation and demodulation. All of the orthogonal subcarriers are transmitted simultaneously. By dividing a wider spectrum into many narrow band subcarriers, a frequency selective fading channel is converted into many flat fading channels over each subcarrier if the subcarrier spacing is small compared to the channel coherence bandwidth. Thus, a simple equalization technique could be used in the receiver to combat the inter-symbol interference. DSRC uses 64 subcarriers where 52 subcarriers are actually used for signal transmission. Out of these 52 subcarriers, 48 are data subcarriers and 4 subcarriers are pilot symbols used for phase tracking. Fig. 1 shows the training sequence structure both in time and frequency. Two long training symbols are across all the subcarriers and 4 pilot subcarriers are only embedded in subcarriers $-21,-7,7,21$.

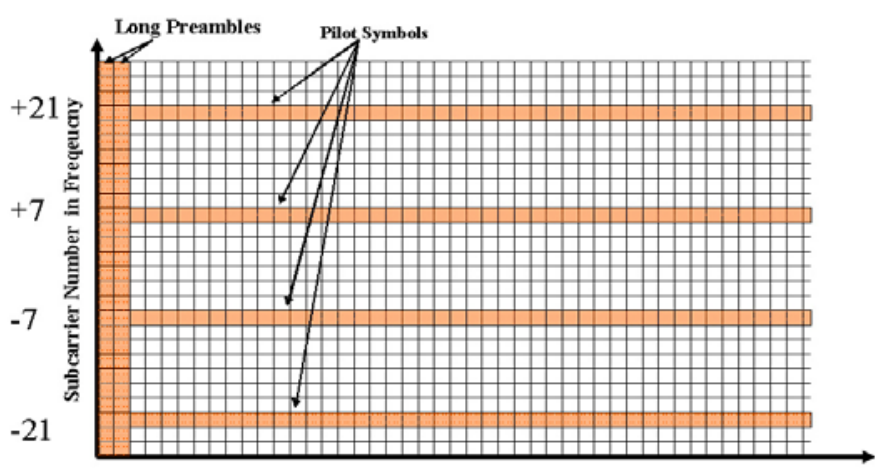

OFDM Symbol Number in Time

Figure 1: Two long preambles and pilot subcarriers in time and frequency

Fig. 2 shows the physical layer data frame structure. A1-A10 are ten identical short training symbols, each is 16 samples long. A subset of these symbols are used for packet detection, automatic gain control(AGC), and various diversity combining schemes. The remaining short training symbols are used for coarse frequency offset estimation and coarse symbol timing estimation. These short training symbols are followed by two long identical training sym- 


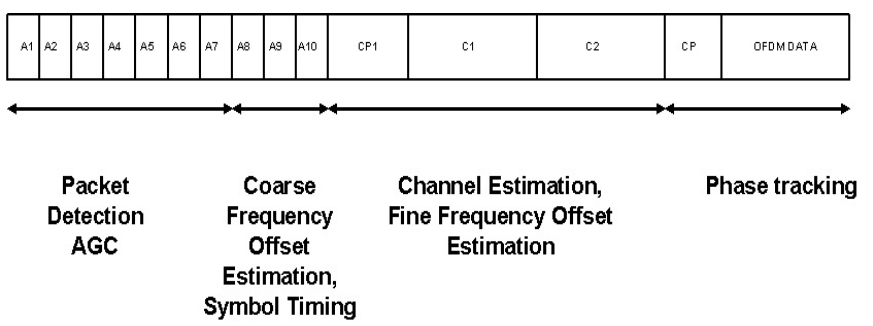

A1-A10 : Ten Short Training Sequence, each 16 samples long

C1-C2: Long Training Sequence, total 160 samples long

CP: Cyclic Prefix, 16 samples long for each OFDM symbol

Figure 2: DSRC PHY Frame Format

bols, C1-C2, which are used for channel estimation, fine frequency and symbol timing estimation. $\mathrm{C} 1$ and $\mathrm{C} 2$ are 64 samples long and the 32-sample long CP1 is the cyclic prefix which protects against intersymbol interference (ISI) from the short training symbols. After short and long training symbols, comes the actual modulated payload OFDM symbols. The first OFDM data symbol is the physical layer header which is BPSK modulated and specifies the modulation scheme used in the payload OFDM symbols that follows. Each OFDM symbol consists of 64 samples and a 16-sample long $\mathrm{CP}$ which is pre-appended for each OFDM symbol to combat ISI. Some of the key physical layer parameters used in DSRC are listed in Table I.

Finally, it is worth noting that IEEE 802.11a is primarily designed for indoor WLAN applications. Thus, all PHY parameters are optimized for the indoor low-mobility propagation environment. Aside from the fact that the DSRC signal bandwidth is $10 \mathrm{MHz}$ (half of the IEEE 802.11a signal bandwidth) in addition to some differences in the transmit power limit, the DSRC PHY follows exactly the same frame structure, modulation scheme and training sequences specified by IEEE 802.11a PHY. However, DSRC applications require reliable communication between OnBoard Units (OBUs) and from OBU to Roadside Unit (RSU) when vehicles are moving up to 120 miles/hour and having communication ranges up to 1000 meters. This environment is drastically different from the indoor low-mobility environment and its implications on the DSRC PHY performance turn out to be non-trivial as demonstrated in the next section.

Table 1: Key Parameters in the DSRC Physical Layer Standard

\begin{tabular}{|l|l|}
\hline Data Rate & $3,4.5,6,9,12,18,24,27 \mathrm{Mbps}$ \\
\hline Modulation & $\begin{array}{l}\text { BPSK, QPSK, 16-QAM, } \\
\text { 64-QAM }\end{array}$ \\
\hline Coding Rates & $1 / 2,2 / 3,3 / 4$ \\
\hline \# Subcarriers & 52 \\
\hline \# Pilot Tones & 4 \\
\hline $\begin{array}{l}\text { OFDM Symbol } \\
\text { Duration }\end{array}$ & $8 \mu \mathrm{sec}$ \\
\hline Guard Interval & $1.6 \mu \mathrm{sec}$ \\
\hline Subcarrier Spacing & $156.25 \mathrm{KHz}$ \\
\hline Signal Bandwidth & $10 \mathrm{MHz}$ \\
\hline
\end{tabular}

\section{DSRC PHYSICAL LAYER PERFORMANCE EVALUATION}

In this section, an implementation of the current DSRC physical layer standard is described and the BER performance is evaluated, under a wide variety of vehicles speeds, using Matlab simulations. ${ }^{1}$ The motivation to conduct a detailed physical layer study of DSRC is multi-fold: first, to evaluate the parameters in the current PHY specification and find out whether we could improve the system performance for outdoor high-speed vehicle environment by modifying some of these parameters; second, to figure out the best packet size and date rate for different applications and third, to generate BER curves necessary for building an integrated PHY-network simulation testbed for VANETs as illustrated in section IV. To meet these objectives, we first discuss the transmitter and receiver baseband processing algorithms used in the simulation. Numerous approaches has been proposed in the literature for designing various aspects of the transmitter and receiver processing blocks and we pick the most robust and frequently used ones. Next, we review the channel models and measurements reported in the literature and utilize them to characterize the DSRC channel parameters at $5.9 \mathrm{GHz}$. Finally, we present the simulation results which confirm the robustness of DSRC against large delay spreads, yet, its sensitivity to high vehicle speeds.

\subsection{DSRC Wireless Link Model}

\subsubsection{DSRC Transmitter}

Fig. 3 illustrates the transmitter processing blocks. Input bit streams are first scrambled using pre-defined random bit sequence, then scrambled data bits are encoded using a 64 state $1 / 2$ rate convolutional code. Higher coding rate is achieved by puncturing from the same $1 / 2$ rate convolutional encoded data bits. Using a single convolutional code at both transmitter and receiver simplifies the encoder and decoder design.

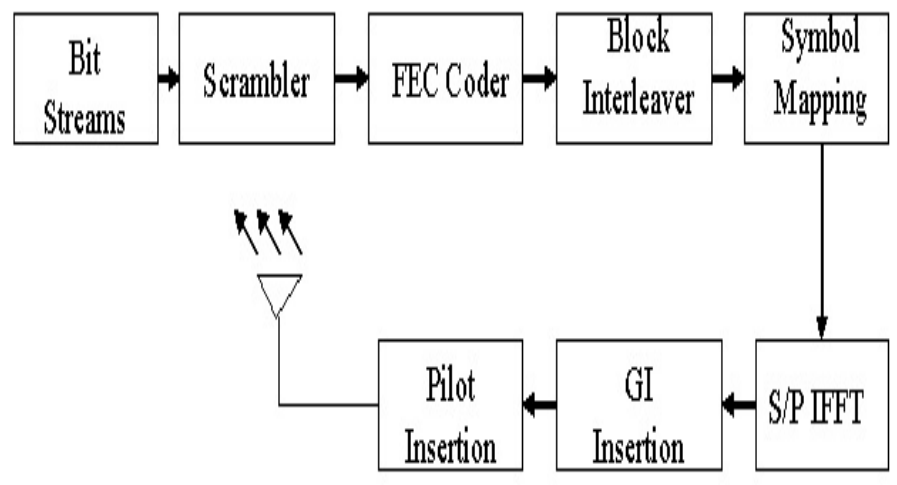

Figure 3: DSRC Transmitter Processing Blocks

After data bits are encoded, they proceed through a block interleaver. The interleaver redistributes the transmitted bits in both time and frequency such that continuous bursty bit errors caused by channel fading would have little impact on the performance of the convolutional decoder. A desirable interleaver pattern depends on the channel characteristics. For instance, using an interleaver under additive white Gaussian noise (AWGN) channel does not im-

\footnotetext{
${ }^{1}$ Although the detailed frame structure and training symbols are specified in the standard, different chip vendors have their own proprietary baseband processing algorithms.
} 
prove the system performance. For a typical indoor operating environment, the channel could be characterized as a slow frequencyselective fading channel [20]. Due to low mobility, the channel coherence time is generally much larger than the packet transmit duration, thus the channel is assumed constant for the duration of a transmitted packet. IEEE 802.11a sets the interleaving depth to one OFDM symbol which, in terms of bit length, depends on the modulation scheme used. Basically, there is no additional benefits by interleaving across multiple OFDM symbols for the indoor environment since the channel is assumed static for contiguous OFDM symbols. On the other hand, for the high mobility outdoor environment, channel coherence time will be much less than that of the indoor environment and turns out to be on the order of the packet length. In this case, the system performance might be improved using a longer interleaver depth, e.g. interleaving across both frequency and different OFDM symbols.

After interleaving, the data bits are mapped into symbols according to different modulation schemes. Inverse Fast Fourier Transform (IFFT) is performed on these modulated symbols, thus data symbols are carried on a set of orthogonal subcarriers. A cyclic prefix (also called Guard Interval) is inserted at the front of each OFDM symbol to combat the ISI introduced by the channel and short and long training preambles are inserted at the beginning of the packet. At this stage, the packet would be ready for transmission.

\subsubsection{Channel Model}

DSRC devices are projected to operate around $5.9 \mathrm{GHz}$ in a high speed mobile outdoor environment with communication ranges up to 1000 meters. However, there are hardly any results available in the open literature on wide-band channel modeling for this operating environment. Extensive studies for narrow-band channel measurements has been conducted in the cellular mobile environment [20]. However, due to the communication range, carrier frequency and signal bandwidth differences, these results can not be directly utilized in characterizing the DSRC channel. Some wideband channel measurements has been gathered for IEEE 802.11 type devices in the indoor low-mobility environment around 5.3 $\mathrm{GHz}[21][22]$. The channel parameters derived from these measurements are significantly different from the DSRC channel in terms of delay spread and Doppler spectrum characteristics.

Channel modeling involves two important aspects: large scale path loss and small scale fading. The former is used to determine the mean received signal power at a particular distance from the transmitter (DSRC applications could also use the path loss model to derive the relative distance between the transmitter and the receiver). On the other hand, small scale fading generally involves the modeling of multi-path fading, power delay profile, and Doppler spectrum. It is worth noting that both large and small scale signal variations have great impact on the packet error rate performance. A generic impulse response of a time-varying wireless channel $h(t, \tau)$ could be expressed as

$$
h(t, \tau)=\sum_{n=0}^{N} \beta_{n}(t) \delta\left(t-\tau_{n}\right)
$$

where $N$ is the number of multiple propagation paths, $\tau_{n}$ is the excess delay of the $n$th path, and $\beta_{n}(t)$ is the complex channel gain associated with $n$th path. Typically $\tau_{n}$ is specified in terms of multiples of the OFDM sample period $T$, and $T$ is the inverse of the signal bandwidth. In DSRC, $T=100 \mathrm{~ns}$. Let $P_{n}$ denote the power of the $n$th path, so $P_{n}=E\left\{\left|\beta_{n}\right|^{2}\right\}$. The channel delay spread, fading statistics, and Doppler spectrum are typically used to fully describe the mobile small scale channel characteristics and channel path loss is taken into account by $\beta_{n}$ in Eq.(1).

Channel measurements are reported in [23] for vehicle-to- vehicle (no mobility) channel at $900 \mathrm{MHz}$ and up to 40 feet separation distance between transmitter and receiver. The root mean square (RMS) delay spread turns out to be around $20 \mathrm{~ns}$ and the Rician $\mathrm{K}$ factor is also computed, yet, it was argued that the fading statistics do not strictly follow the Rician model. Instead, a strong line of sight (LOS) component combined with a strong reflected wave from the road way might be more appropriate to model the short range vehicle-to-vehicle communication channel [23]. Path loss and delay spread measurements for vehicle-to-vehicle communication are also reported in [24]. In this experiment, two vehicles having a fixed 10 to 30 meters separation are moving together in and out of a tunnel, and the RMS delay spread was found to vary from $10 \mathrm{~ns}$ to $40 \mathrm{~ns}$.

In [25], a wide-band outdoor channel measurement campaign at $5 \mathrm{GHz}$ has been conducted in urban, suburban, and rural areas. The transmit antenna is held at fixed locations at heights 4,12 , and 45 meters respectively and the receiver antenna is mounted on the rooftop of a slowly moving vehicle. The measurement distances are within 30-300 meters and path loss exponents within 1.4-3.5 in LOS and 2.8-5.9 for non-LOS are reported. The mean excess delay and mean RMS delay spread are typically within 29-102 ns and 22-88 ns respectively. The scenario where the transmit antenna was held at 4 meters could be useful to characterize the communication channel between the OBU and RSU in DSRC. Delay spread measurements results at $1.8 \mathrm{GHz}$ in urban environments are also reported in [28]. Transmit antenna heights of 5, 7 and 10 meters are used in the testing and the range is up to 600 meters. Mean RMS delay spread was found to range from $20 \mathrm{~ns}$ to $70 \mathrm{~ns}$ depending on the location of the street and maximum RMS delay spread reaches levels up to $512 \mathrm{~ns}$. Larger RMS delay spread usually correlates to the intersections. Path loss measurement in residential areas at $5.8 \mathrm{GHz}$ is also conducted in [32] and the results could also be used in vehicle to RSU communications.

A narrow band measurement of signal fading statistics and Doppler spectrum had been conducted in [27] for a vehicle-to-vehicle communication ranging from 30-300 meters. A Rician distribution was found to fit best to the measured received signal power in a least square sense and this conclusion is mainly due to fact that a strong LOS is mostly present during the testing. Doppler spectrum is triangular shaped and there is a strong peak at DC frequency.

Various theoretical analysis of Doppler spectrum for mobile to mobile communication could be found in [30][29] [31]. Under the assumption of 3-D multi-path scattering power density functions, generic antenna radiation pattern, and absence of a deterministic component, the Doppler spectrum for a mobile-to-mobile radio channel is derived in [30] as a function of $V 1 / V 2$, where $V 1$ and $V 2$ are the vehicle speeds of the transmitter and receiver. Also traditional 2-D Jake's spectrum [36] is a special case of this 3-D model. In [29], a statistical mobile-to-mobile channel model is also derived based on the assumption of Gaussian process of the channel and level crossing rate and duration of the fade are computed based on the proposed model.

Practical Doppler spectrum measurements and theoretical investigation had been conducted in [31] for different operating environments: urban, suburban, and rural. There are three types of Doppler spectrum that occur often on each tap of the channel impulse response: the "horned" spectrum, the narrow spectrum, and the flat spectrum. The horned spectrum arises when the received signal in the azimuth plane has a wide range of angle of arrivals. The narrow spectrum happens when the scattering is over a narrow 
range of angles in the azimuth plane. The flat spectrum occurs when both the scattering and the receiver's antenna patterns are isotropic in three dimensions. In typical vehicle-to-vehicle communications the horned and the narrow Doppler spectrum will be present with high probability since the wave propagates mainly in the azimuth plane between transmitter and receiver. In particular, the narrow spectrum usually happens in the first or second tap of the channel impulse response when there is strong LOS between transmitter and receiver.

In summary, various studies and measurements results in the literature reveal that the DSRC channel will exhibit different characteristics under different operating environments. For vehicle-tovehicle communications in the LOS scenario, RMS delay spread would be less than $50 \mathrm{~ns}$ which translates to at most two delay taps in Eq.(1) since DSRC sample period $T=100 \mathrm{~ns}$. For a relatively short range communication in LOS scenario, the direct path and the reflected path from the ground will not be resolvable from the receiver hardware point of view, and these two paths will be combined into one single complex channel gain. For a longer communication range in a non-LOS scenario, worst case RMS delay spread reported could be up to 400ns and in this scenario substantial multipath and high vehicle speeds will have a dramatic impact on the bit error rate of the system. The DSRC channel model adopted in our simulations is described in section IV.B.

\subsubsection{DSRC Receiver}

Fig.4 shows the DSRC receiver baseband processing blocks. After the carrier sensing circuit declares sufficient RF energy present in the received signal, the first step in the baseband processing is the packet detection. Packet detection is carried out using the delay and correlate method in [33]. In essence, this method takes advantage of periodicity of the ten short training sequences. A normalized cross correlation between received signal and its delayed version is computed, and the delay is 16 samples long which is the period of the ten short training sequences. This cross correlation value is continuously computed using sliding window until enough number of cross correlation values exceed the threshold for reliable packet detection.

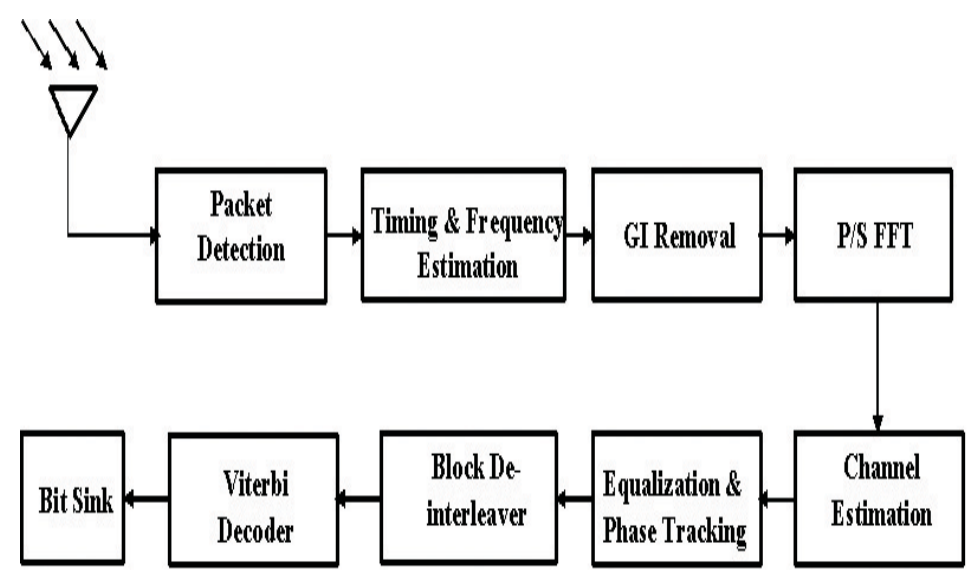

Figure 4: DSRC Receiver Baseband Processing Blocks

After the packet is detected, fine timing and frequency synchronization is performed using the long training sequence. The objective of fine timing estimation is to find the starting point of FFT operation on the following received OFDM symbols. The fine timing estimation method is based on the search for the maximum cross correlation value between received long training sequence and the actual long training sequence. Due to the multi-path, noise and interference, the fine timing estimator itself is a random variable around its true mean value. It's better to shift this mean value towards inside the range of cyclic prefix rather than to delay it into OFDM symbol since if the timing estimation falls inside the OFDM symbol, it will give wrong FFT window for further processing.

Due to the symbol clock difference between transmitter and receiver and the Doppler frequency present in the DSRC system, frequency offset needs to be estimated from the long training sequence. OFDM system is sensitive to the carrier frequency offset since nonzero carrier frequency offset will cause inter-carrier interference. The effect of frequency offset on the SNR is studied in [34]. In DSRC devices, the required symbol clock accuracy should be less than $20 \mathrm{ppm}$, and this translates to about $117 \mathrm{KHz}$ maximum frequency offset introduced in the received signal. The maximum Doppler frequency that will be present in the DSRC system is about $2 \mathrm{KHz}$ assuming the maximum relative speed of 240 miles per hour. Compared to the carrier frequency and the maximum symbol clock drifts, it could be ignored. Utilizing the two identical long training sequences, the frequency offset estimation could be based on the averaging of the phase difference between a received signal sample and its delayed version over many received signal samples [35]. The more samples are used in the averaging the better the quality of the frequency offset estimation.

After the received signal is corrected by the estimated frequency offset, it is transformed to frequency domain using FFT. Channel estimation is done by averaging of the computed complex channel gain of each subcarrier using the two long training sequences. Basically, a simple division of the received long training signal after FFT and the original long training sequence yields the estimated channel gain. Residual frequency offset and carrier phase offset estimation is achieved with the aid of the pilot subcarrier inserted between data subcarriers. In DSRC, vehicles could move at very high speeds, and the channel might change within a packet duration. Thus, phase tracking errors might cause the processing of higher modulation schemes to perform poorly. In order to remedy this potential problem, a new pilot design is proposed and evaluated in [8], where pilot subcarriers both in time and frequency are used to perform better phase tracking. However, the drawback of using the new scheme is the processing delay and increased complexity of the algorithms.

Afterwards, the received signal is mapped into soft bits and deinterleaved. Finally, soft bits information are passed into Viterbi decoder, CRC check is performed on the decoded bits to determine whether there is bit error in the packet or not.

\subsection{DSRC PHY Simulation Results}

Current DSRC physical layer has been simulated in details using Matlab and we follow the specifications for training sequences, scrambling sequences, convolutional encoder, and interleaver in the DSRC ASTM E2213-03 Standard [2]. Packet error rate measurements using Atheros baseband chip sets have also been conducted to verify our baseband simulation results in the AWGN scenario.

In Fig. 5, the DSRC channel model used in the simulation is depicted, where $\beta_{n}$ and $\tau_{n}$ are defined in Eq. (1). We assume 400 ns RMS delay spread for the multi-path environment along with an exponentially decaying power delay-profile in the simulation. Jakes' Doppler spectrum [36] is used to generate fading taps under various vehicle speeds.

Fig. 6 shows the bit error rate versus SNR for a DSRC receiver at $12 \mathrm{Mbps}$ which is the direct outcome of using 16-QAM modulation scheme along with $1 / 2$ rate convolutional coding. The date rate in DSRC ranges from $3 \mathrm{Mbps}$ to $27 \mathrm{Mbps}$, and hence, this set of 


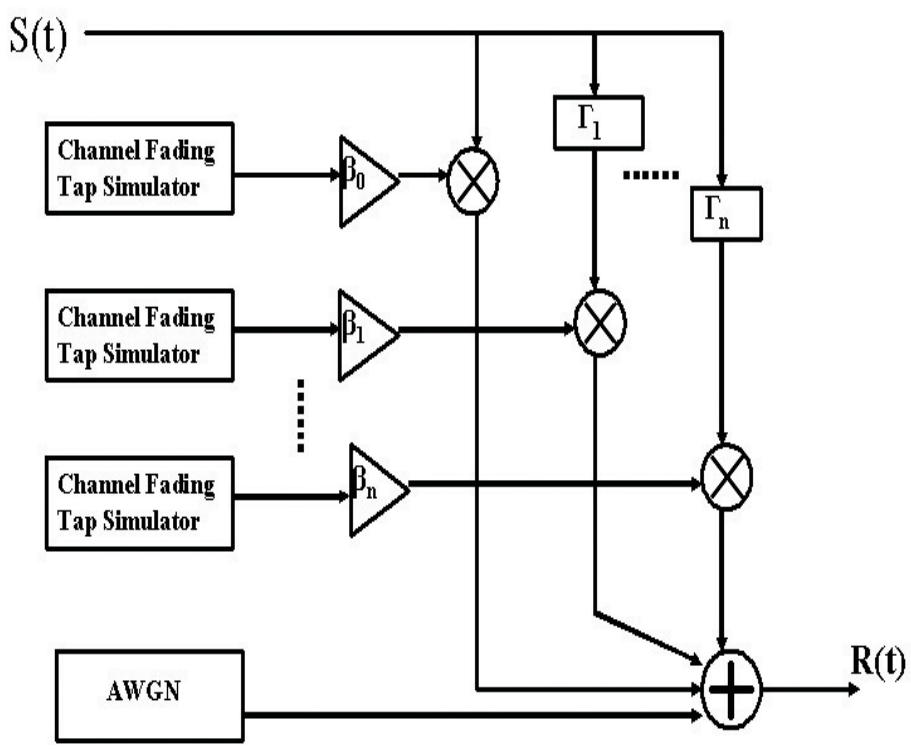

Figure 5: DSRC Channel Simulator

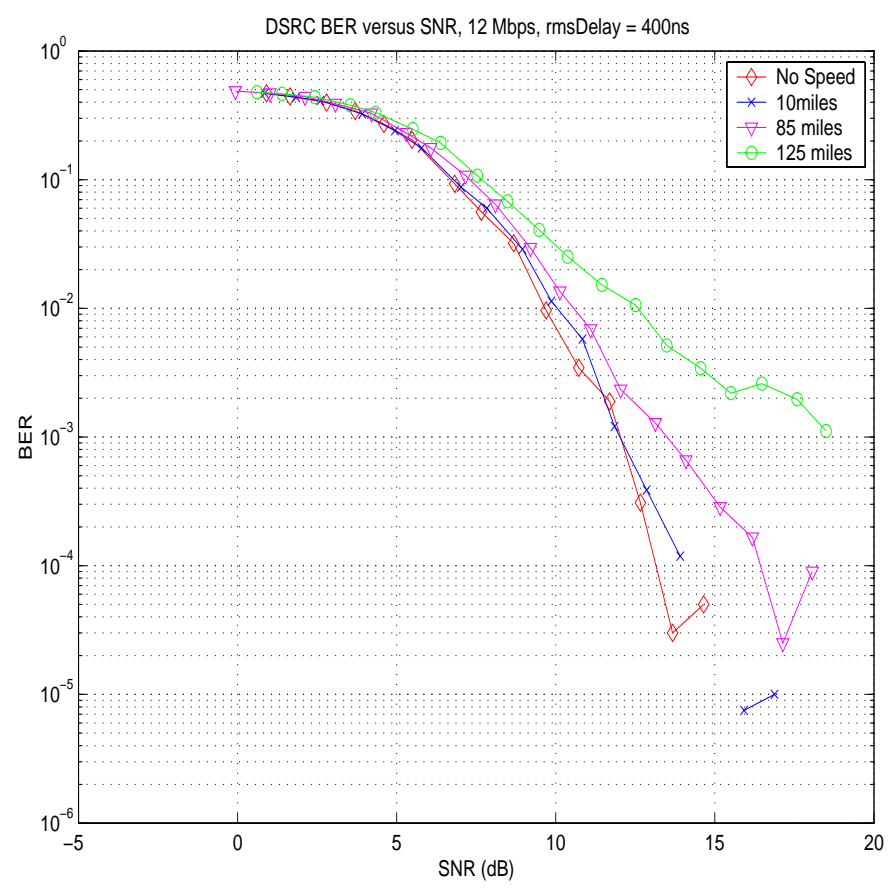

Figure 6: BER versus SNR at 12 Mbps and 400ns RMS delay spread experiments employ a medium data rate of $12 \mathrm{Mbps}$ as a simulation example. For more reliable communication, the lowest modulation scheme (BPSK) with $1 / 2$ rate code could be used. Data rates and packet sizes depend primarily on the QoS requirements dictated by various applications. It can be noticed from Fig. 6 that DSRC could tolerate very large delay spreads due to the very long cyclic prefix (1600 ns), yet, the BER is highly sensitive to vehicle speeds, especially for a higher modulation scheme. As vehicles move at higher speeds, there will be an irreducible error floor present no matter what the SNR is. This is attributed to the fact that channel estimation is done using training sequence at the beginning of the packet and then used for the rest of the packet. When vehicles move at high speeds, the channel characteristics will change substantially within a packet duration and, therefore, the current training sequence structure might not be sufficient to support high mobility outdoor applications.

\section{VEHICLE COLLISION AVOIDANCE OVER DSRC: FEASIBILITY STUDY}

In this section, we develop a simulation testbed, using Qualnet simulator, for vehicular ad hoc networks based on the current DSRC PHY/MAC protocol stack, realistic vehicle mobility models and vehicle collision avoidance safety applications. In the next section, we describe the network and application models underlying this study. In the following section, we present the VCA throughput and latency simulation results.

\subsection{VANET Simulation Setup}

In this section, we outline the simulation parameters and modeling assumptions adopted for: i) The DSRC vehicular ad hoc network and ii) The application running on top of this network, namely vehicle collision avoidance. As pointed out earlier, a detailed physical layer model is required for accurate network simulation. Physical layer performance does not only affect the absolute performance of a protocol, it can even change the relative ranking among the protocols for the same scenario since its impact on different protocols may be non-uniform [37]. There are generally two methods used for physical layer modeling in network simulations, namely SNR threshold based and BER based. Under the former method, the receiver accepts the packet when the computed SNR value is above the SNR threshold for a particular modulation scheme. On the other hand, the method based on BER decides whether or not a packet is received successfully based on the packet length and bit error rate deduced by the pre-computed BER versus SNR curve for every modulation scheme at the receiver. It has been concluded in [37] that the BER method is considered more realistic and accurate than the one based on SNR threshold. Therefore, we adopt the BER based method throughout this study.

Next, we outline the parameters underlying our simulation testbed. We consider a mobile ad hoc network that consists of 100 vehicles moving on a city road map of dimensions $6600 \mathrm{X} 4200$ meters as shown in Fig. 7. This road topology is ported from the CORSIM (CORridor SIMulation) vehicle traffic simulator [38]. Furthermore, the mobility model adopted in this paper is ported from CORSIM and converted to the Qualnet simulator format in order to reflect realistic mobility patterns of vehicles on the roads. The maximum vehicle speed was set to about 75 miles per hour. We also assume that vehicles are equipped with omni-directional antennas that can radiate fixed and equal transmission power of $17 \mathrm{dBm}$ which translates to a transmission range of approximately 300 meters as recommended by VSCC for VCA applications [19]. As dictated by the standard, the DSRC receiver sensitivity is set to $-77 \mathrm{dBm}$. The 
maximum transmission power supported by the DSRC radio is 28.5 $\mathrm{dBm}$ which translates to a range of approximately 1000 meters. Each data point on the graphs represents the average of three independent simulation runs with different random seeds. Each simulation run is carried out for the duration of 100 seconds. According

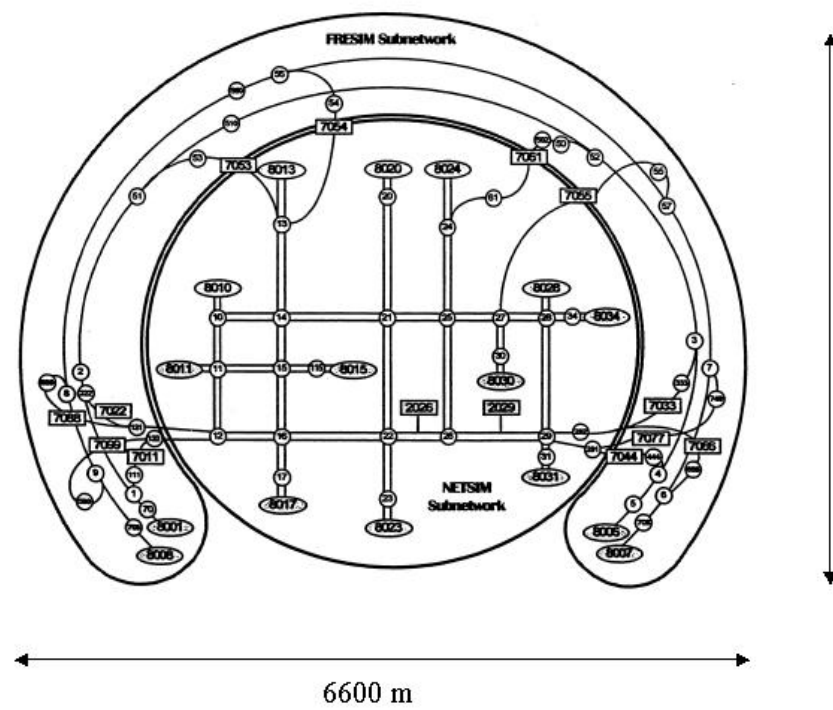

Figure 7: Road map used in DSRC VANET simulations

to the design guidelines and performance requirements provided in [19], the VCA application is modeled as a single-hop broadcast application. Thus, a new application model has been added to Qualnet to model the class of VCA applications where each vehicle periodically broadcasts a VCA packet of fixed size (100/200 bytes) as a collision warning to its single-hop neighbors. Therefore, multi-hop routing is not needed in this study since the traffic pattern generated by the application of interest is single-hop broadcast transmissions. The network load is varied via gradually increasing the VCA packet generation rate per vehicle $(\lambda)$ from 1 packets/sec to 10 packets/sec. Finally, we assume that this application is operating over a single channel.

\subsection{Results and Discussion}

The prime objective of this paper is to quantify the performance of vehicle collision avoidance applications on top of DSRC VANETs. This is of paramount importance to: i) Assess the level of support that the current DSRC standard draft provides for safety applications and ii) Further guide the standard development in order to meet the QoS requirements dictated by safety applications. The performance metrics used in this study is the VCA packet throughput and latency. The VCA throughput is defined as the long-run average percentage of single-hop neighbors who successfully receive the VCA broadcast packet. Notice that node B is considered a neighbor of node $\mathrm{A}$ if it lies within node A's radio transmission range (300 meters in this study). The importance of this measure stems from the fact that it reflects the degree of DSRC PHY/MAC support for single-hop broadcast traffic which could be encountered in a wide variety of safety applications [19]. On the other hand, the VCA latency is defined as the long-run average time elapsed between sending a VCA packet at the source node and successfully receiving that packet at a single-hop neighbor. This measure is critical from a safety perspective since it should be less than $100 \mathrm{msec}$ in order to constitute a valid warning for a distracted driver driving at high speed and at few meters distance from the vehicles ahead [19]. Notice that these two measures are computed at the end of each simulation run.

In Fig. 8, the VCA latency is plotted versus the network load which is varied from 1 to 10 packets per second per node. We have conducted separate simulation experiments for examining two different packet sizes, namely 100 and 200 bytes. The observations captured from this set of results are multi-fold. First, the VCA delay is noticed to gradually increase with the range of network loads of interest in this study as intuition might suggest. This is due to the potential overlapping, in time and space, among the broadcast events of spatially close vehicles which causes frequent backoffs due to the physical carrier sensing of the wireless channel [3]. ${ }^{2} \mathrm{Sec}-$ $4200 \mathrm{n} p$ nd, we notice that the same trend holds for the two VCA packet sizes examined in this study. Moreover, we notice that varying the VCA packet size has slight impact on performance. In fact, the scenario of 100 byte VCA packet outperforms the scenario of 200 byte VCA packet only by a factor of $15 \%$ on the average. This is attributed to the fact that the VCA packet latency is dominated by the backoff mechanism in CSMA/CA compared to the considerably smaller transmission delay which is in the order of fraction of microseconds for the given data rates and packet sizes. Finally, it is worth noting that the delay performance falls well within the $100 \mathrm{msec}$ delay requirement for safety applications over the shown range of packet generation rates. Thus, we conclude the current standing of DSRC provides promising latency support for timecritical safety applications.

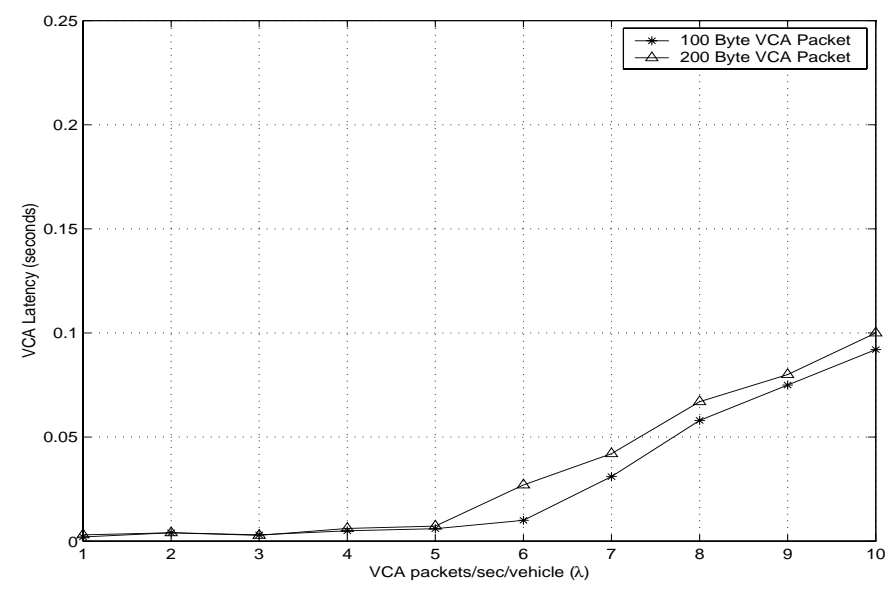

Figure 8: VCA Packet Latency

In Fig. 9, the VCA throughput is plotted versus the network load. It is straightforward to observe that, once again, varying the VCA packet size has slim impact on the application throughput; the 100 byte VCA packet case outperforms the 200 byte case only by a factor less than $6 \%$ on the average. In addition, the VCA packet throughput slightly decreases within the range of network loads of interest to safety applications. Finally, we observe that the average VCA throughput, under both experiments, lies between $50 \%$ and $60 \%$, which is considered modest performance from a safety point of view since more than $40 \%$ of vehicles, on the average, within the

\footnotetext{
${ }^{2}$ Notice that CSMA/CA does not provide packet collision avoidance through virtual carrier sensing (i.e. RTS/CTS/ACK) for broadcast packets generated under the class of VCA applications.
} 
sender's broadcast range will not receive the VCA warning messages. Therefore, we strongly advocate the need for a QoS requirement on the VCA throughput, which parallels the aforementioned $100 \mathrm{msec}$ latency requirement proposed by VSCC. Both requirements should constitute the driving force for guiding the DSRC protocol development towards tight coupling to safety applications performance.

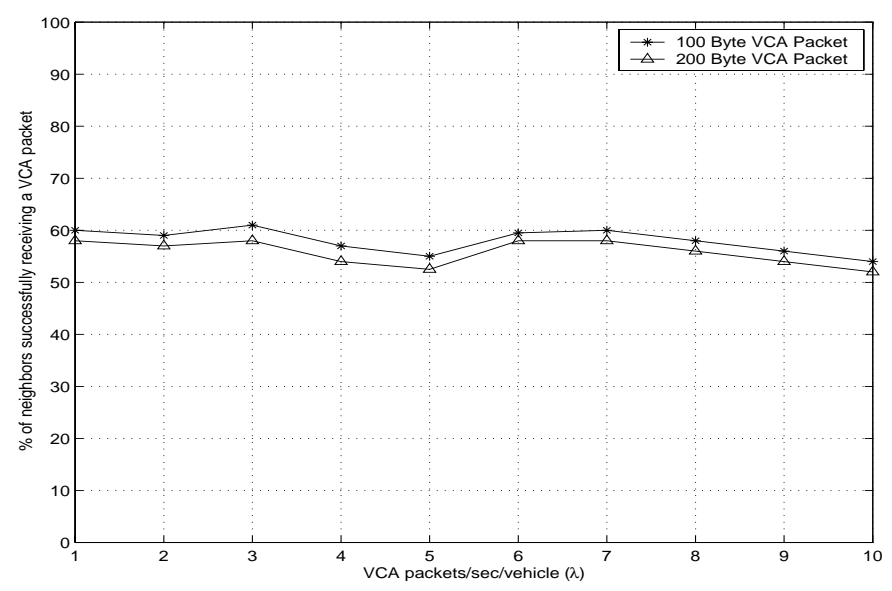

Figure 9: VCA Packet Throughput

\section{CONCLUSIONS}

In this paper we investigated the performance of vehicle collision avoidance applications on top of DSRC vehicular ad hoc networks. The study proceeded through two phases. In the first part of the paper, we quantified the bit error rate performance of DSRC wireless links under a wide variety of mobility scenarios. Simulation results reveal the immunity of DSRC PHY layer to large delay spreads, yet, significant performance degradation is noticed under high mobility scenarios. In the second part of the paper, we conducted an integrated PHY-network simulation study for DSRC vehicular ad hoc networks adopting realistic mobility models and executing single-hop broadcast applications as a representative of vehicle collision avoidance. We observed that DSRC achieves favorable VCA latency performance compared to the application requirements. On the other hand, the VCA throughput turns out to be moderate which motivates further improvements, possibly via exploiting the multi-channel capability of DSRC. Extending this work to asses the performance of other types of safety and nonsafety applications (e.g. toll collection and file transfer) is a potential avenue for future work. Finally, it is imperative to develop empirical channel models based on real measurements that reveal an accurate representation of the DSRC wireless link behavior in the $5.9 \mathrm{GHz}$ band.

\section{REFERENCES}

[1] http://www.onstar.com

[2] Standard Specification for Telecommunications and Information Exchange Between Roadside and Vehicle Systems - 5GHz Band Dedicated Short Range Communications (DSRC) Medium Access Control (MAC) and Physical Layer (PHY) Specifications, ASTM E2213-03, Sep. 2003.

[3] IEEE 802.11 WG, Part 11: Wireless LAN Medium Access Control (MAC) and Physical Layer (PHY) Specifications, IEEE, August 1999.
[4] P. Varaiya, "Smart Cars on Smart Roads: Problems of Control," IEEE Transactions on Automatic Control, vol. 38, No. 2, pp. 195-207, Feb. 1993.

[5] J. Zhu and S. Roy, "MAC for Dedicated Short Range Communications in Intelligent Transport System," IEEE Communications Magazine, pp. 60-67, Dec. 2003.

[6] Q. Xu, R. Sengupta and D. Jiang "Design and Analysis of Highway Safety Communication Protocol in $5.9 \mathrm{GHz}$ Dedicated Short Range Communication Spectrum," Proc. of IEEE Vehicular Technology Conference-Spring, April 2003.

[7] X. Yang, J. Liu, F. Zhao and N. Vaidya "A Vehicle-to-Vehicle Communication Protocol for Cooperative Collision Warning," Technical Report. University of Illinois at Urbana-Champaign, Dec. 2003.

[8] S. Sibecas, C. Corral, S. Emami and G. Stratis, "On the Suitability of 802.11a/RA for High-Mobility DSRC," Proc. IEEE Vehicular Technology Conference, Oct. 2002.

[9] D. Lee et al., "A Wireless Token Ring Protocol for Intelligent Transportation Systems," Proc. IEEE Intelligent Transportation Systems Conference, Aug. 2001.

[10] S. Bana and P. Varaiya, "Space Division Multiple Access (SDMA) for Robust Ad hoc Vehicle Communication Networks," Proc. IEEE Intelligent Transportation Systems Conference, Aug. 2001.

[11] L. Michael and M. Nakagawa, "Multi-hopping Data Considerations for Inter-vehicle Communication over Multiple Lanes," Proc. IEEE Vehicular Technology Conference, Oct. 1997.

[12] L. Briesemeister, L. Schafers and G. Hommel,"Disseminating Messages among Highly Mobile Hosts based on Inter-vehicle Communications," Proc. IEEE Intelligent Vehicles Symposium, Oct. 2000.

[13] H. Hartenstein et al., "Position-Aware Ad Hoc Wireless Networks for Inter-Vehicle Communications: the Fleetnet Project," Proc. ACM Mobihoc, Oct. 2001.

[14] M. Mauve, J. Widmer, and H. Hartenstein, "A Survey on Position-based Routing in Mobile Ad Hoc Networks," IEEE Network, pp. 30-39, Nov./Dec. 2001.

[15] M. Lott et al., "Medium Access and Radio Resource Management for Ad hoc Networks based on UTRA TDD,' Proc. ACM Mobihoc, Oct. 2001.

[16] http://www.netlab.nec.de/Projects/fleetnet.htm

[17] Notice of Proposed Rulemaking and Order FCC 02-302, Federal Communications Commission, Nov. 2002.

[18] Notice of Proposed Rulemaking and Order FCC 03-324, Federal Communications Commission, Feb. 2003.

[19] Task 3: Identify Intelligent Vehicle Safety Applications Enabled by DSRC - Interim Report, Vehicle Safety Communications Consortium, Jan. 2003.

[20] T.S. Rappaport (Ed.), Wireless Communications: Principle and Practice. Prentice-Hall, New Jersey, 1996.

[21] R. V. Nee, and R. Prasad, OFDM For Wireless Multimedia Communications, Artech House Publishers, 2002.

[22] J. Terry and J. Heiskala, OFDM Wireless LANs: A Theoretical and Practical Guide. Sams Publishing, Indiana, Dec. 2001.

[23] J. S. Davis, and Jean Paul M. G. Linnartz, "Vehicle to Vehicle RF propagation Measurements," 1994 Conference Record of the Twenty-Eighth Asilomar Conference ,Volume: 1, Oct. 1994.

[24] A. V. Batista da Silva, and M. Nakagawa, "Radio wave propagation measurements in tunnel entrance environment for 
intelligent transportation systems applications," 2001 IEEE Intelligent Transportation Systems Conference Proceedings, Oakland, CA, August 25-29, 2001.

[25] X. Zhao, J. Kivinen, P. Vainikainen, and K. Skog, "Propagation Characteristics for Wideband Outdoor Mobile Communications at $5.3 \mathrm{GHz}$," IEEE Journal On Selected Areas In Communications, Vol. 20, No. 3, April 2002.

[26] J. P. Singh, N. Bambos, B. Srinivasan, and D. Clawin, "Wireless LAN performance Under Varied stress Conditions in Vehicular Traffic Scenarios," Vehicular Technology Conference, 2002. Proceedings. VTC 2002-Fall., Volume: 2 , 24-28 Sept. 2002

[27] J. Maurer, T. Fgen, W. Wiesbeck, "Narrow-band measurement and analysis of the inter-vehicle transmission channel at $5.2 \mathrm{GHz}$,' IEEE Vehicular Technology Conference, 2002.

[28] A. A. Arowojolu, A.M.D. Turkmani, and J. D. Parsons, "Time dispersion measurements in urban microcellular environments," IEEE 44th Vehicular Technology Conference, 8-10 June, 1994.

[29] A. S. Akki, "Statistical properties of mobile-to-mobile land communication channels," IEEE Trans. on Vehicular Technology, Vol. 43, No. 4, November 1994.

[30] F. Vatalaro, A. Forcella, "Doppler spectrum in mobile-to-mobile communications in the presence of three-dimensional multipath scattering," IEEE Trans. on Vehicular Technology, Vol. 46, No. 1, February 1997.
[31] X. Zhao, J. Kivinen, P. Vainikainen, K. Skog, "Characterization of Doppler spectra for mobile communications at $5.3 \mathrm{GHz}$," IEEE Trans. Vehicular Tecnology, Vol. 52, No. 1, January 2003.

[32] G. Durgin, T. S. Rappaport, and H. Xu, "Measurements and Models for Radio Path Loss and Penetration Loss In and Around Homes and Trees at $5.85 \mathrm{GHz}$," IEEE Transactions On Communications, Vol. 46, No. 11, November, 1998.

[33] T. M. Schmidl, and D. C. Cox, "Low-Overhead, Low-Complexity Burst Synchronization for OFDM," IEEE International Conference on Communications, Vol. 3, 1996.

[34] T. Pollet, M. V. Bladel, and M. Moeneclaey, "BER Sensitivity of OFDM Systems to Carrier Frequency Offset and Wiener Phase Noise," IEEE Transactions on Communications, Vol. 43, 1995.

[35] J-J. Van De Beek, M. Sandell, P. O. Borjesson, "ML Estimation of Time and Frequency offset in OFDM Systems," IEEE Transactions on Signal Processing, Vol. 45, No. 7, July 1997.

[36] W. C. Jakes, Microwave Mobile Communications, New York, Wiley, 1974.

[37] M. Takai, J. Martin, R. Bagrodia "Effects of Wireless Physical Layer Modeling in Mobile Ad Hoc Networks," IEEE Journal On Selected Areas In Communications, Vol. 21, No. 3, April, 2003.

[38] J. Joines et al., "Traffic Flow Simulation Using CORSIM," Proc. Winter Simulation Conference, Dec. 2000. 